\title{
Research on Reflection and Rotation Features of Binomial Coefficient Distributions
}

\author{
Zhu Minghan ${ }^{1}$, Zheng Jeffrey Zhijie ${ }^{2, *}$ \\ ${ }^{1}$ School of Software, Yunnan University, Kunming, China \\ ${ }^{2}$ Yunnan Laboratory of Quantum Information, Kunming, China \\ Email address: \\ crystalj000@163.com (Zhu Minghan), conjugatelogic ayahoo.com (Zheng Jeffrey Zhijie) \\ ${ }^{*}$ Corresponding author
}

To cite this article:

Zhu Minghan, Zheng Jeffrey Zhijie. Research on Reflection and Rotation Features of Binomial Coefficient Distributions. Science Discovery. Vol. 7, No. 4, 2019, pp. 239-248. doi: 10.11648/j.sd.20190704.21

Received: July 8, 2019; Accepted: August 22, 2019; Published: August 27, 2019

\begin{abstract}
With the rapid development of frontier neural network, artificial intelligence and big data technology, the related theory and application research of combinatorial mathematics are more and more extensive. The application of binomial coefficient in combinatorial mathematics is an effective way to solve the research of information coding and quantum computation. Binomial coefficients and its distributions are the core topic in probability statistics, and there are many theories and applications related to them.In this paper, the quantification of binomial coefficients and the characteristic of reflection rotation transformation are studied using three-dimensional diagrams. Using variant construction, the combinatorial clustering properties are investigated applying binomial formulas and sample distributions and their combinatorial patterns are illustrated. It is proved that the basic binomial coefficient formula and its extended model have obvious properties of reflection and rotation invariance.
\end{abstract}

Keywords: Binomial Coefficient, Spatial Extension, Variant Construction, Reflection, Rotation

\section{二项式系数量化分布的反射旋转特性研究}

\section{朱明涵 ${ }^{1}$, 郑智捷 ${ }^{2 *}$}

1云南大学软件学院, 昆明, 中国

云南省量子信息重点实验室, 昆明, 中国

\section{邮箱}

crystalj000@163.com (朱明涵), conjugatelogic@yahoo.com（郑智捷）

摘要：随着前沿神经网络, 人工智能, 大数据技术的快速发展, 组合数学的相关理论和应用研究越来越广泛。二项式 系数在组合数学中的应用是解决信息编码及量子计算研究的有效途径。二项式系数及其分布是概率统计中的核心论题, 已有丰富的理论及其应用与其关联。本文利用一类基础二项式系数公式，通过变换参数的模式，形成与反射和旋转关 联的作用。通过三维散点图示对其二项式系数的量化情况及反射旋转变换特性进行研究, 展现了利用变值测量所形成 的二项式公式的潜在扩展能力。从整体不变性的角度, 系统地研究与这类二项式公式及其扩展模式伴随的各种组合聚 类性质。证明该类基础二项式系数公式及其扩展模式具有明显的反射和旋转不变特性。

关键词：二项式系数, 扩展模式, 变值测量, 反射性, 旋转不变性 


\section{1. 引言}

在数学与计算机科学领域, 随着前沿神经网络, 人工 智能, 大数据技术的快速发展, 组合数学的相关理论和应 用研究变得越来越重要 [1]。对二项式系数公式的量化特征 研究得到了越来越多的数学家, 大数据人工智能专家的关 注[2]。

二项式系数与帕斯卡三角形存在着一一对应的联系。 从不变性的角度, 古今中外的数学家们对此产生浓厚的兴 趣。除了推导公式, 恒等式证明, 寻找特殊数字排列规律 等研究之外, 利用二项式系数公式进行可视化分析对前沿 大数据, 云计算等学科尤为重要。

利用计算机图形学和图像处理技术的可视化技术, 是 一类将数据转换成图形或图像在屏幕上显示出来, 再进行 交互处理的理论、方法和技术[3]。涉及到计算机图形学、 图像处理、计算机视觉、虚拟现实技术、计算机辅助设计 等多个领域, 成为研究数据表示、数据处理、决策分析等 一系列解决与大数据, 人工智能相关核心问题的综合技术。

对于可视化技术[4]的一个重要分支: 科学计算可视化, 把科学数据, 包括测量获得的数值、图像或是复杂系统涉 及到的数字信息变为直观的图像信息表示。使伴随时间和 空间变化的物理现象或物理量直观地呈现在研究者面前, 以便于观察、模拟和计算 [5]。

利用组合方法给出特定数学, 组合恒等式的证明是组 合数学研究的热点 [6]。在物理学, 数学, 计算机科学领域 中, 二项式系数起到重要作用, 表现出的旋转反射不变性 为其核心特性。[7]本文扩展形成的二项式公式族, 所形成 的分布具有明显的反射旋转不变性。期待该类不变特性能 够与量子力学、经典物理学、数学、计算机科学、卷积神 经网络中的不变特性密切结合, 对各类应用计算方法中的 优化研究有帮助。

本文利用变值测量模型和方法, 结合三维可视化分析 二项式系数公式量化分布特点, 对其不同参数族形成的反 射旋转和特定特征进行分析总结。从全体不变的角度, 系 统地考察二项式系数及其扩展表示的各种组合聚类性质。

\section{2. 理论基础}

\section{1. 二项式系数与组合数}

二项式系数 [8](binomial coefficient): 或组合数, 定义 为形如展开后 $\mathrm{x}$ 的系数 (其中 $\mathrm{n}$ 为自然数, $\mathrm{k}$ 为整数)。

组合数 [9](combinatorial number): 从 $\mathrm{n}$ 个不同元素中取 出 $m(m \leq n)$ 个元素所有组合的个数, 即从 $n$ 个不同元素中取 出 $\mathrm{m}$ 个元素的组合数。用符号 $\left(\begin{array}{l}n \\ m\end{array}\right)$ 表示。

\section{2. 变值编码体系}

2010年创建的变值体系 [10]包括三个部分: 变值逻辑, 变值测量和变值可视化。变值逻辑是基于 $0-1$ 向量模式的 新型逻辑体系。
该体系在传统向量状态的基础上扩展两类向量运算: 置换和互补, 将传统的 $\mathrm{n}$ 元逻辑函数空间从 $2^{2^{n}}$ 函数空间扩 展成为 $2^{n} ! * 2^{2^{n}}$ 配置函数空间 [11]。变值测量和变值图示 已应用于不同的问题, 包括: 经典密码序列分析, 量子密 码序列检测, 染色体全基因序列图示, 心电图信号检测, 蝙蝠信号处理等[10]。

本文主要部分扩展在变值测量中形成的基础二项式 公式。

\section{3. 反射旋转不变性}

反射变换 [12](reflection transformation)是平面几何中 一种重要变换, 将平面上的轴反射变换, 镜面反射变换都 称为反射变换。

作为仿射变换[13], 可以描述图像或者图形之间的多 种变换模式, 其中包含平移, 旋转, 反射等。

旋转不变性[14]: 平面几何中, 当中点坐标确定之后, 旋转算符转动 360 度与原图重合。在离散几何应用中, 90 度旋转最为常见 $[15]$ 。本文旋转运算限于 90 度旋转, 不涉 及到其他任意角度的旋转运算。

\section{3. 处理流程}

\section{1. 二项式系数公式由来}

在变值体系专著[10]P39-50中, 四个测度的有序结构 $\{a, b, c, d\}$, 在置换和组合两种运算下共有192种表示结构。 任意的一个结构都对应一组二项式系数。

在本文中, 选择基础二项式公式为: $f(m, p, k)=\left(\begin{array}{l}m-p \\ k\end{array}\right)\left(\begin{array}{l}p \\ k\end{array}\right)$, 取值范围为 $0 \leq \mathrm{p} \leq \mathrm{m} ; 0 \leq \mathrm{k} \leq \mathrm{p} ;$ $\mathrm{k} \leq \mathrm{m}-\mathrm{p}$ 。

当 $m=10$ 时, 系数项分布在 $11 * 11$ 的 $1 / 4$ 区域中, 其余 $3 / 4$ 区域为 0 。组成如图 1 所示的等腰三角形。

\begin{tabular}{|c|c|c|c|c|c|c|}
\hline$f(10, p, k)$ & $\begin{array}{lllll}0 & 1 & 2 & 3 & 4\end{array}$ & 5 & 67 & 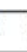 & 80 & 10 \\
\hline 10 & \\
\hline 9 & & & & & & \\
\hline 8 & & & & & & \\
\hline 7 & & & & & & \\
\hline 6 & & & & & & \\
\hline 5 & \multicolumn{6}{|c|}{1} \\
\hline 4 & 15 & 25 & 15 & & & \\
\hline 3 & 3580 & 100 & 8035 & & & \\
\hline 2 & 286390 & 100 & & & & \\
\hline 1 & 9162124 & 25 & 2421 & & 6 & \\
\hline 0 & $\begin{array}{lllll}1 & 1 & 1 & 1 & 1\end{array}$ & 1 & 11 & & & \\
\hline$k$ & & & & & & \\
\hline
\end{tabular}

图 $1 \mathrm{~m}$ 取 10 基础二项式系数项三角形分布。

根据该二项式系数公式, 利用其反射旋转不变的特性, 变换不同的取值范围, 可得出不同的二项式系数公式。且 每组二项式系数公式, 取值范围各不相同。处理过程如图 2所示。 


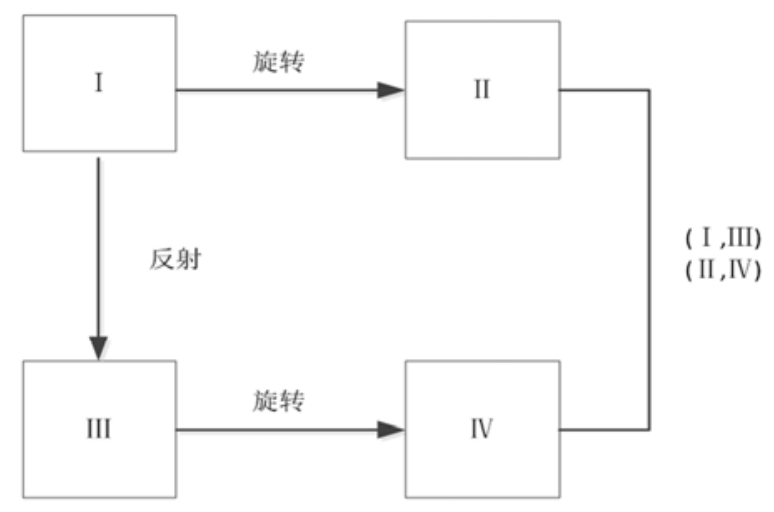

图2 二项式系数公式处理流程。

首先从基本二项式系数公式 $f(m, p, k)=\left(\begin{array}{l}\mathrm{m}-\mathrm{p} \\ k\end{array}\right)\left(\begin{array}{l}p \\ k\end{array}\right)$, 取值范围为 $0 \leq_{\mathrm{p}} \leq_{\mathrm{m} ; 0} \leq_{\mathrm{k}} \leq_{\mathrm{p} ; \mathrm{k}} \leq_{\mathrm{m}-\mathrm{p}}$, 三维作图得到 区域 I, 经过反射相应位置二项式系数值f, 得到图 3 中区 域III范围, 推断出区域III对应的二项式系数公式为 $f_{3}(m, p, k)=\left(\begin{array}{l}\mathrm{m}-\mathrm{k} \\ p\end{array}\right)\left(\begin{array}{l}k \\ p\end{array}\right)$, 相应的取值范围为 $0 \leq{ }_{\mathrm{k}} \leq \mathrm{m} ; 0 \leq$ $\mathrm{p} \leq_{\mathrm{k} ; \mathrm{k}} \leq_{\mathrm{m}-\mathrm{p}}$ 。

由于 I 和III区域与 II 和 IV 区域存在着旋转不变性的 特性, 因此由 I 和III区域旋转得到 II 和IV区域, 则以区域 I 和区域III的二项式系数公式可以得出区域 II 和 IV 的范 围及表达式为: $f_{2}(m, p, k)=\left(\begin{array}{l}\mathrm{p} \\ m-k\end{array}\right)\left(\begin{array}{l}m-p \\ m-k\end{array}\right), 0 \leq_{\mathrm{p}} \leq_{\mathrm{m}}$;

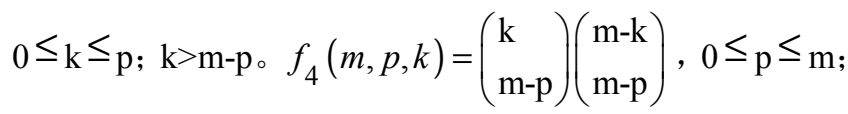
$0 \leq_{\mathrm{k}} \leq_{\mathrm{p}} ; \mathrm{k}>\mathrm{m}-\mathrm{p}$ 。

四个区域的二项式系数以 $\mathrm{m}-\mathrm{p}=\mathrm{k}$ 和 $\mathrm{p}=\mathrm{k}$ 的射线, 呈现 对称结构。

如图3所示, 系统描述了每个公式取值范围的变化情 况, 以及得出每个二项式公式的过程。

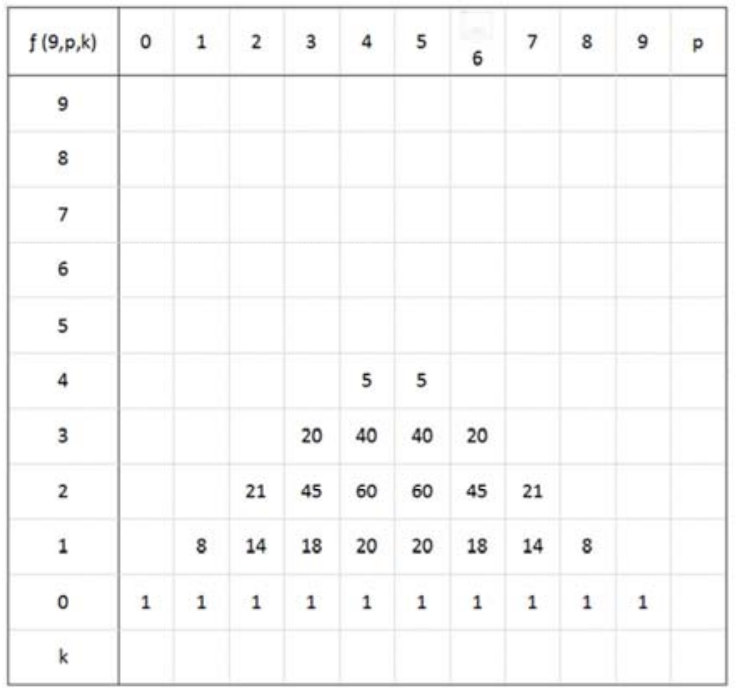

(a)

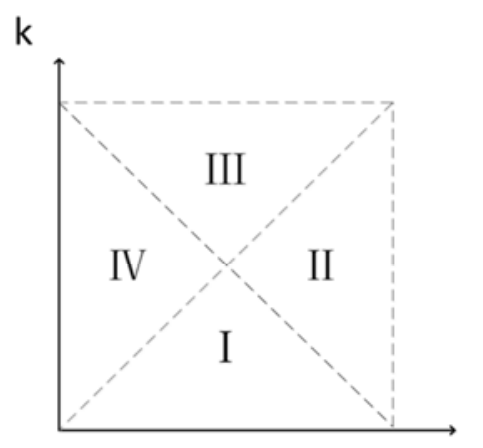

p

图3 二项式及扩展公式反射旋转变换过程。

I : $f(m, p, k)=\left(\begin{array}{l}m-p \\ k\end{array}\right)\left(\begin{array}{l}p \\ k\end{array}\right), 0 \leq \mathrm{p} \leq \mathrm{m} ; 0 \leq \mathrm{k} \leq \mathrm{p} ;$ $\mathrm{k} \leq \mathrm{m}-\mathrm{p}$;

III: $f_{3}(m, p, k)=\left(\begin{array}{l}\mathrm{m}-\mathrm{k} \\ p\end{array}\right)\left(\begin{array}{l}k \\ p\end{array}\right), 0 \leq_{\mathrm{k}} \leq_{\mathrm{m}} ; 0 \leq_{\mathrm{p}} \leq_{\mathrm{k}} ;$ $\mathrm{k} \leq \mathrm{m}-\mathrm{p}$;

II $: f_{2}(m, p, k)=\left(\begin{array}{l}\mathrm{k} \\ \mathrm{m}-\mathrm{p}\end{array}\right)\left(\begin{array}{l}\mathrm{m}-\mathrm{k} \\ \mathrm{m}-\mathrm{p}\end{array}\right), 0 \leq \mathrm{p} \leq \mathrm{m} ; 0 \leq_{\mathrm{k}} \leq_{\mathrm{p}} ;$ $\mathrm{k}>\mathrm{m}-\mathrm{p} ;$

$\mathrm{IV}: f_{4}(m, p, k)=\left(\begin{array}{l}\mathrm{p} \\ m-k\end{array}\right)\left(\begin{array}{l}m-p \\ m-k\end{array}\right), 0 \leq_{\mathrm{k}} \leq_{\mathrm{m} ; 0} \leq_{\mathrm{p}} \leq$ $\mathrm{k} ; \mathrm{k}>\mathrm{m}-\mathrm{p}$ 。

\section{2. 二项式系数公式矩阵图示}

用矩阵结构表示基础二项式系数公式 $f(m, p, k)=\left(\begin{array}{l}m-p \\ k\end{array}\right)\left(\begin{array}{l}p \\ k\end{array}\right)$ 。对比 $\mathrm{m}$ 取值为奇数和偶数的二项 式系数分布情况。 $\mathrm{m}$ 取值为 9 和 10 的系数项分布情况如图 4(a)-(b)所示。

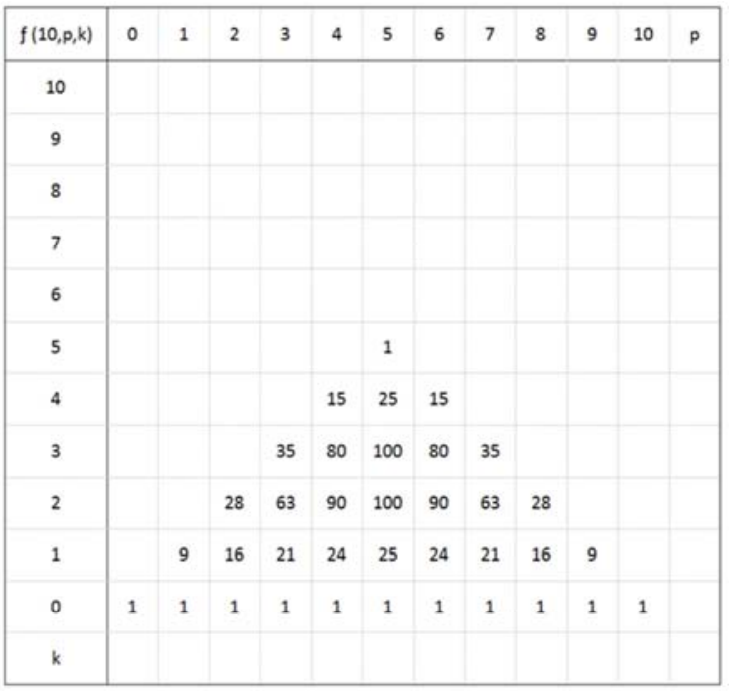

(b)

图4 基础二项式系数公式矩阵图示 (a)m=9 (b)m=10。 
在 经过反射 变 换 后 形 成的二项式公式 $f_{2}(m, p, k)=\left(\begin{array}{l}\mathrm{k} \\ \mathrm{m}-\mathrm{p}\end{array}\right)\left(\begin{array}{l}\mathrm{m}-\mathrm{k} \\ \mathrm{m}-\mathrm{p}\end{array}\right), 0 \mathrm{pm} ; 0 \mathrm{kp} ; \mathrm{k}>\mathrm{m}-\mathrm{p}$ 。以 $\mathrm{m}=9$

\begin{tabular}{|c|c|c|c|c|c|c|c|c|c|c|c|}
\hline$f(9, p, k)$ & 0 & 1 & 2 & 3 & 4 & 5 & 6 & 7 & 8 & 9 & $p$ \\
\hline 9 & 1 & 1 & 1 & 1 & 1 & 1 & 1 & 1 & 1 & 1 \\
\hline 8 & & 8 & 14 & 18 & 20 & 20 & 18 & 14 & 8 & \\
\hline 7 & & & 21 & 45 & 60 & 60 & 45 & 21 & & \\
\hline 6 & & & & 20 & 40 & 40 & 20 & & & \\
\hline 5 & & & & & 5 & 5 & & & & \\
\hline 4 & & & & & 5 & 5 & & & & \\
\hline 3 & & & & 20 & 40 & 40 & 20 & & & \\
\hline 2 & & & 21 & 45 & 60 & 60 & 45 & 21 & & \\
\hline 1 & & 8 & 14 & 18 & 20 & 20 & 18 & 14 & 8 & \\
\hline 0 & 1 & 1 & 1 & 1 & 1 & 1 & 1 & 1 & 1 & 1 \\
\hline$k$ & & & & & & & & & \\
\hline
\end{tabular}

(a)
和 $m=10$ 为例, 对比 $m$ 奇偶取值中系数分布情况。如图 $5(\mathrm{a})-(\mathrm{b})$ 所示。

图5 基础二项式系数公式反射变换后矩阵图示 (a)m=9 (b)m=10。

最后通过其旋转不变的特性, 对 $\mathrm{f}$ 函数和 $\mathrm{f} 3$ 函数做旋转变换, 得到如图6(a)-(b)所显示的扩展二项式系数分布情况。

\begin{tabular}{|c|c|c|c|c|c|c|c|c|c|c|c|c|}
\hline$f(9, p, k)$ & 0 & 1 & 2 & 3 & 4 & 5 & 6 & 7 & 8 & 9 & $p$ \\
\hline 9 & 1 & & & & & & & & & 1 & \\
\hline 8 & 1 & 8 & & & & & & & 8 & 1 & \\
\hline 7 & 1 & 14 & 21 & & & & & 21 & 14 & 1 & \\
\hline 6 & 1 & 18 & 45 & 20 & & & 20 & 45 & 18 & 1 & \\
\hline 5 & 1 & 20 & 60 & 40 & 5 & 5 & 40 & 60 & 20 & 1 & \\
\hline 4 & 1 & 20 & 60 & 40 & 5 & 5 & 40 & 60 & 20 & 1 & \\
\hline 3 & 1 & 18 & 45 & 20 & & & 20 & 45 & 18 & 1 & \\
\hline 2 & 1 & 14 & 21 & & & & & 21 & 14 & 1 & \\
\hline 1 & 1 & 8 & & & & & & 8 & 1 & \\
\hline 0 & 1 & & & & & & & & 1 & \\
\hline$k$ & & & & & & & & & & & \\
\hline
\end{tabular}

(a)

\begin{tabular}{|c|c|c|c|c|c|c|c|c|c|c|c|c|}
\hline$f(10, p, k)$ & 0 & 1 & 2 & 3 & 4 & 5 & 6 & 7 & 8 & 9 & 10 & $p$ \\
\hline 10 & 1 & & & & & & & & & & 1 & \\
\hline 9 & 1 & 9 & & & & & & & & 9 & 1 & \\
\hline 8 & 1 & 16 & 28 & & & & & & 28 & 16 & 1 & \\
\hline 7 & 1 & 21 & 63 & 35 & & & & 35 & 63 & 21 & 1 & $\mid$ \\
\hline 6 & 1 & 24 & 90 & 80 & 15 & 15 & 80 & 90 & 24 & 1 & \\
\hline 5 & 1 & 25 & 100 & 100 & 25 & 1 & 25 & 100 & 100 & 25 & 1 & \\
\hline 4 & 1 & 24 & 90 & 80 & 15 & & 15 & 80 & 90 & 24 & 1 \\
\hline 3 & 1 & 21 & 63 & 35 & & & & 35 & 63 & 21 & 1 & \\
\hline 2 & 1 & 16 & 28 & & & & & 28 & 16 & 1 & \\
\hline 1 & 1 & 9 & & & & & & 9 & 1 \\
\hline 0 & 1 & & & & & & & & 1 & \\
\hline$k$ & & & & & & & & & & & \\
\hline
\end{tabular}

(b)

图6 二项式系数公式旋转变换后矩阵图示 (a) $m=9$ (b) $m=10$ 。

基础二项式系数公式及其扩展模式的系数分布情况 用如图7(a)-(b)所示的矩阵结构表示。

通过观察图 7 (a), 当 $m=9$ 时, 经过反射旋转后, 数值 与数值之间呈现对称结构, 不存在中心点, 以中间为 5 得 数值形成的两行两列的对称结构。以黄色区域突出显示。 如图7(b), 当 $m=10$ 时, 经过反射旋转后呈现中心对称, 组成 $11 * 11$ 的正方形。最外圈都为 1 , 存在中心点, 且中心 点也为 1 。以中间点为 1 的数值形成一行一列的对称结构。 以黄色区域突出显示。 


\begin{tabular}{|c|c|c|c|c|c|c|c|c|c|c|c|c|}
\hline$f(9, p, k)$ & 0 & 1 & 2 & 3 & 4 & 5 & 6 & 7 & 8 & 9 & $p$ \\
\hline 9 & 1 & 1 & 1 & 1 & 1 & 1 & 1 & 1 & 1 & 1 & \\
\hline 8 & 1 & 8 & 14 & 18 & 20 & 20 & 18 & 14 & 8 & 1 & \\
\hline 7 & 1 & 14 & 21 & 45 & 60 & 60 & 45 & 21 & 14 & 1 & \\
\hline 6 & 1 & 18 & 45 & 20 & 40 & 40 & 20 & 45 & 18 & 1 & \\
\hline 5 & 1 & 20 & 60 & 40 & 5 & 5 & 40 & 60 & 20 & 1 & \\
\hline 4 & 1 & 20 & 60 & 40 & 5 & 5 & 40 & 60 & 20 & 1 & \\
\hline 3 & 1 & 18 & 45 & 20 & 40 & 40 & 20 & 45 & 18 & 1 & \\
\hline 2 & 1 & 14 & 21 & 45 & 60 & 60 & 45 & 21 & 14 & 1 & \\
\hline 1 & 1 & 8 & 14 & 18 & 20 & 20 & 18 & 14 & 8 & 1 & \\
\hline 0 & 1 & 1 & 1 & 1 & 1 & 1 & 1 & 1 & 1 & 1 & \\
\hline$k$ & & & & & & & & & & & \\
\hline
\end{tabular}

(a)

\begin{tabular}{|c|c|c|c|c|c|c|c|c|c|c|c|c|c|}
\hline$f(10, p, k)$ & 0 & 1 & 2 & 3 & 4 & 5 & 6 & 7 & 8 & 9 & 10 & $p$ \\
\hline 10 & 1 & 1 & 1 & 1 & 1 & 1 & 1 & 1 & 1 & 1 & 1 & \\
\hline 9 & 1 & 9 & 16 & 21 & 24 & 25 & 24 & 21 & 16 & 9 & 1 & \\
\hline 8 & 1 & 16 & 28 & 63 & 90 & 100 & 90 & 63 & 28 & 16 & 1 & \\
\hline 7 & 1 & 21 & 63 & 35 & 80 & 100 & 80 & 35 & 63 & 21 & 1 & \\
\hline 6 & 1 & 24 & 90 & 80 & 15 & 25 & 15 & 80 & 90 & 24 & 1 & \\
\hline 5 & 1 & 25 & 100 & 100 & 25 & 1 & 25 & 100 & 100 & 25 & 1 & \\
\hline 4 & 1 & 24 & 90 & 80 & 15 & 25 & 15 & 80 & 90 & 24 & 1 & \\
\hline 3 & 1 & 21 & 63 & 35 & 80 & 100 & 80 & 35 & 63 & 21 & 1 & \\
\hline 2 & 1 & 16 & 28 & 63 & 90 & 100 & 90 & 63 & 28 & 16 & 1 & \\
\hline 1 & 1 & 9 & 16 & 21 & 24 & 25 & 24 & 21 & 16 & 9 & 1 \\
\hline 0 & 1 & 1 & 1 & 1 & 1 & 1 & 1 & 1 & 1 & 1 & 1 & \\
\hline$k$ & & & & & & & & & & & & \\
\hline
\end{tabular}

(b)

图7 基础二项式系数公式及其扩展模式矩阵图示 (a)m=9 (b)m=10。

\section{4. 结果显示}

在三维立体散点表示下:

利用测量图解, 可视化分析并讨论组合系数中不同数 值的分布情况, 变量映射由二项式系数数组作为样本。

二项式系数公式: $f(m, p, k)=\left(\begin{array}{l}m-p \\ k\end{array}\right)\left(\begin{array}{l}p \\ k\end{array}\right)$, 取值范围 为: $0 \mathrm{pm} ; 0 \mathrm{kp} ; \mathrm{km}-\mathrm{p}$ 。

当 $m$ 取值为 9 时, 通过观察图 $8(a)-(b)$, 在 $10 * 10$ 的区域 内总共存在 30 个点, 数值分布呈现对称特征。颜色从蓝色 到红色依次增强。俯视图8(a)能明显观察到最高层有两个 点, 切数值相等, 不存在最高点。俯视图 $8(\mathrm{~d})$ 则展现出最 高点的分布情况。

当 $m=9$ 和 $m=10$ 时, 基础二项式公式三维可视化结果如 下图8(a)-(d)所示。

当 $\mathrm{m}$ 取值为奇数时, 俯视图三角顶部不存在顶点, 最 高为两个数值点。当 $\mathrm{m}$ 取值为偶数时, 俯视图三角形存在 最高顶点。

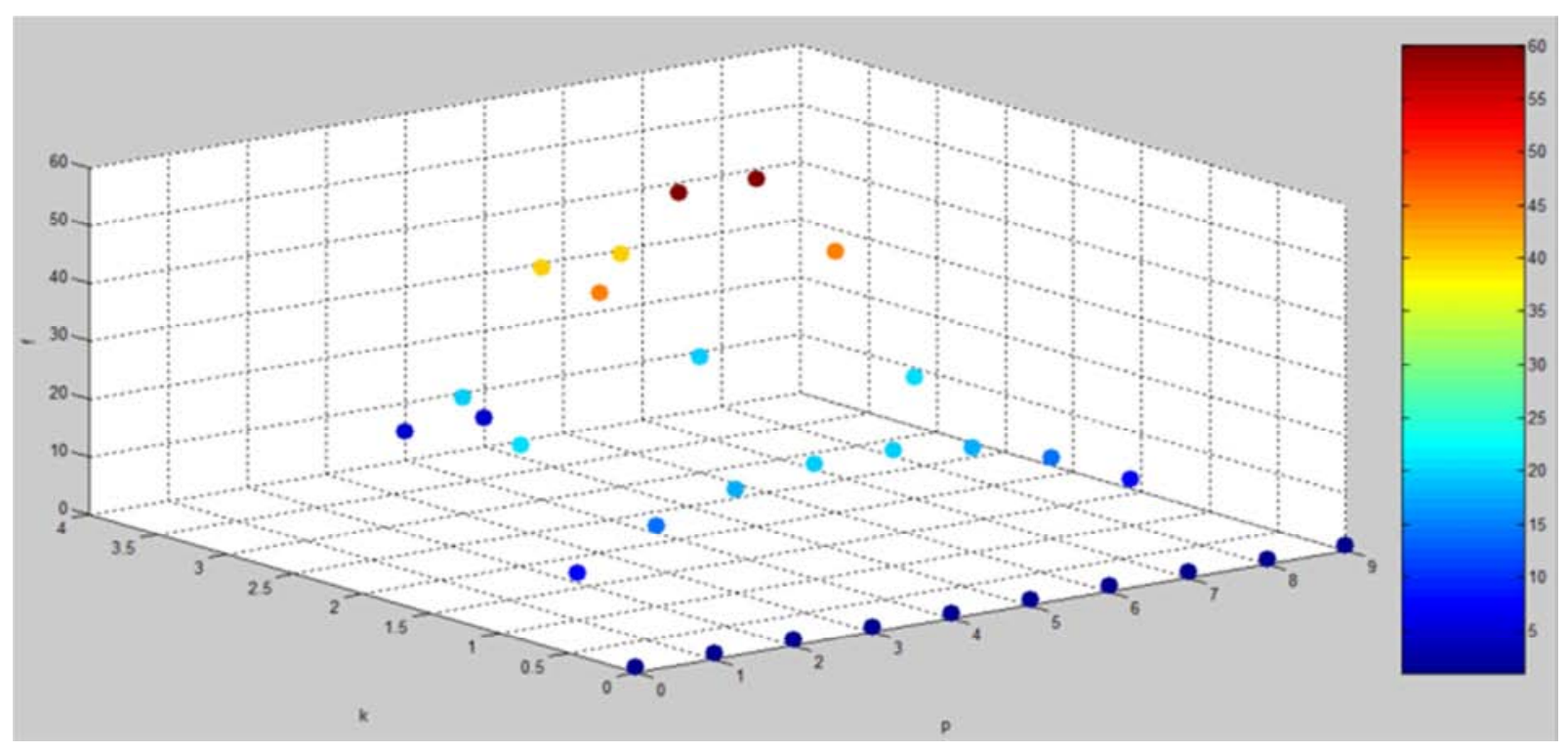

(a) 


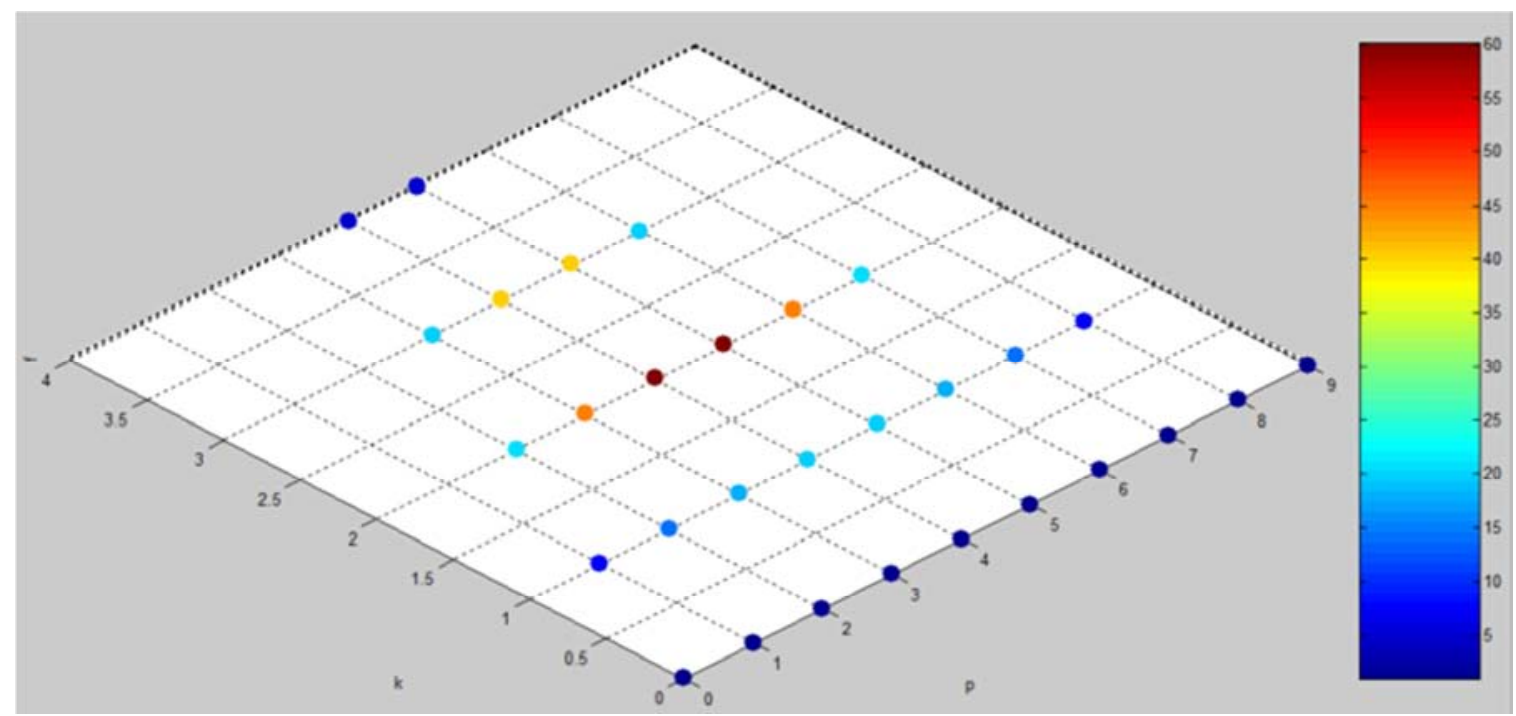

(b)

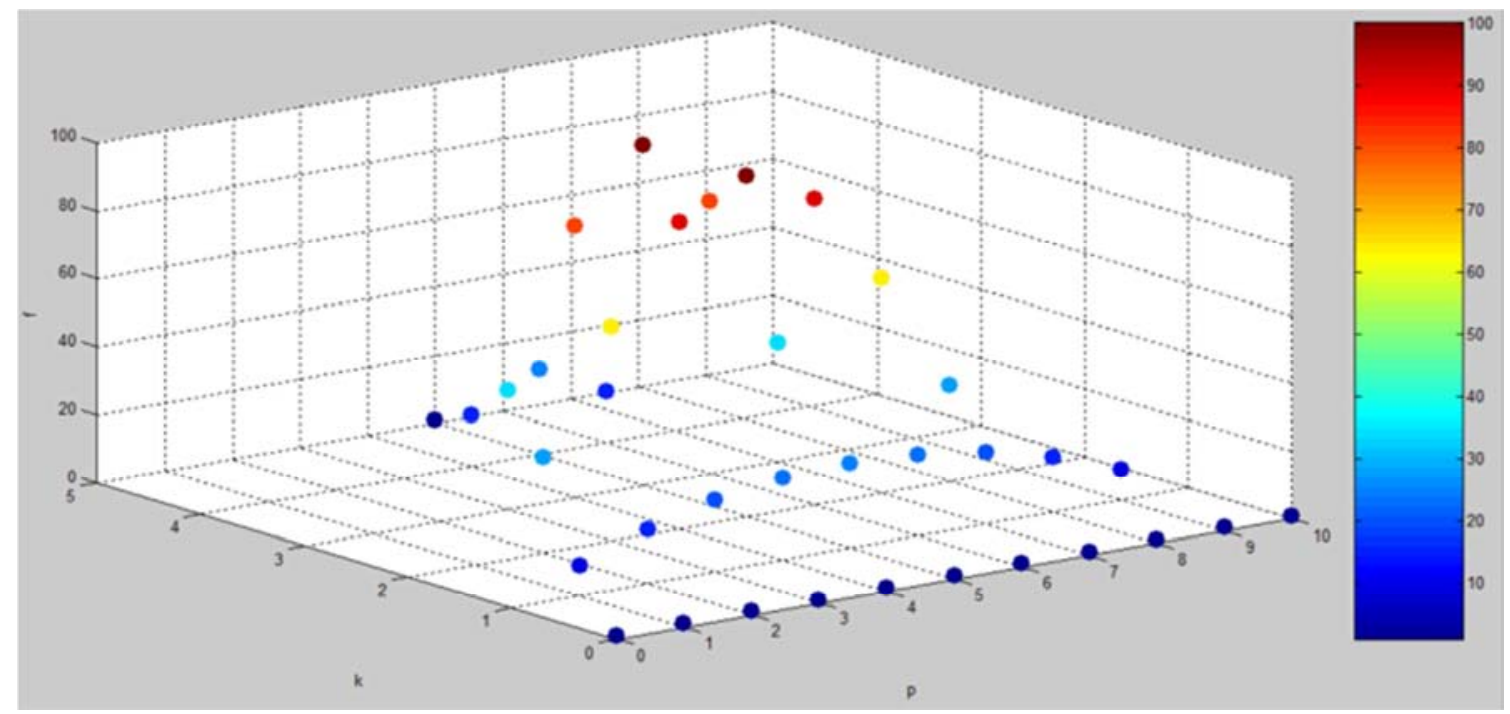

(c)

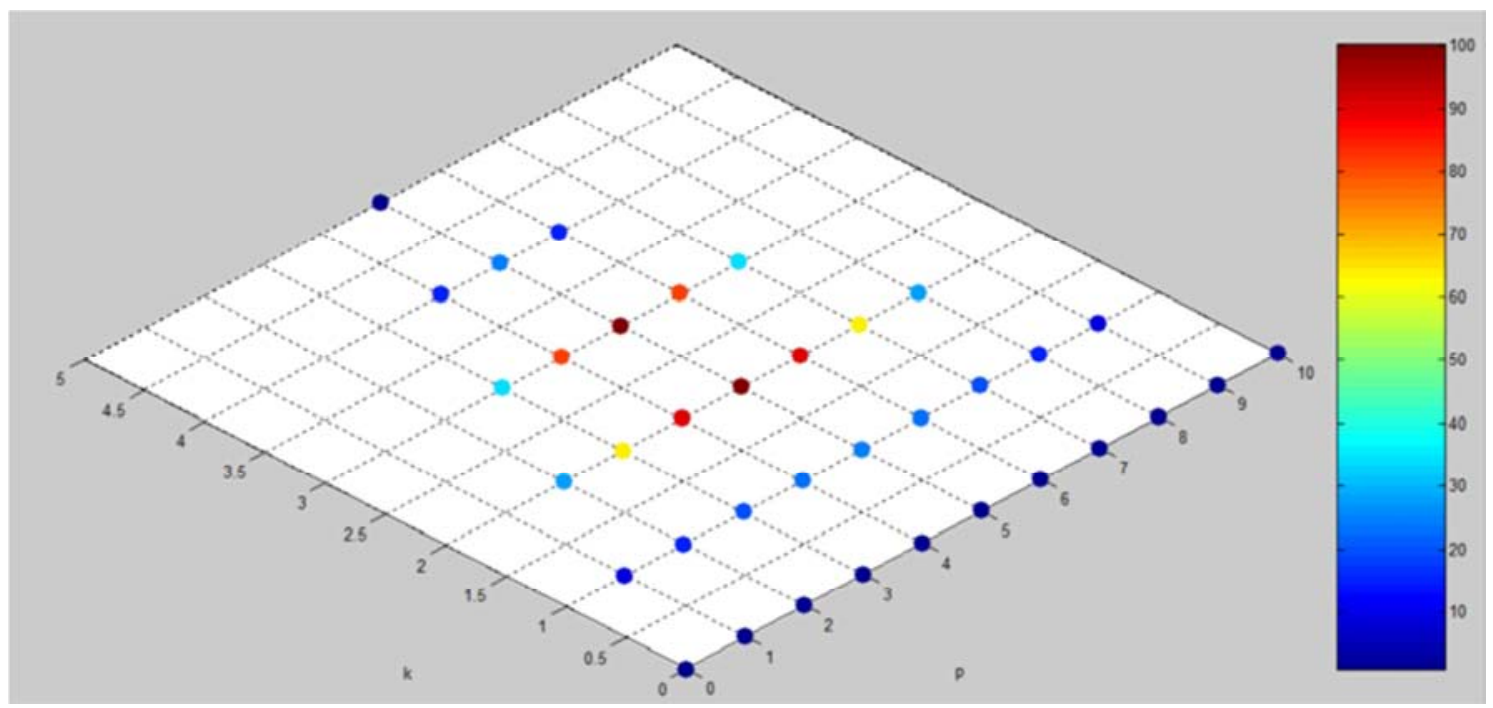

(d)

图8 二项式系数公式三维可视化图示 (a)m=9正视图 (b)m=9俯视图 (c)m $=10$ 正视图 (d)m=10俯视图。 
当 $m$ 取 79和 80 时, 通过观察图 10(a)和图 10(c), 发现 该二项式公式呈现一个峰波。以值为颜色区别, 从深蓝 色到深红色依次增强。底面数值全为 1 , 数值分布以平 面为单位, 颜色依次变强, 数值也相应增加, 且每一层 的数值都呈现出先减小后增大的趋势, 符合对称性原理 及正态分布原则。蓝色数值集中在波峰的下半部分, 红 色集中在峰顶。 俯视图图10(b)和图10(d) 呈现三角形, 中心取值最大, 依次向四周扩散, 取值逐渐减小, 符合帕斯卡三角形法则。 当 $m=79$ 时, 俯视图最高层为两个点, 且两点数值相等。 而 $\mathrm{m}=80$ 时, 只存在一个最高点。

当 $m=79$ 和 $m=80$ 时, 基础二项式公式三维可视化结果 如下图10(a)-(d)所示。

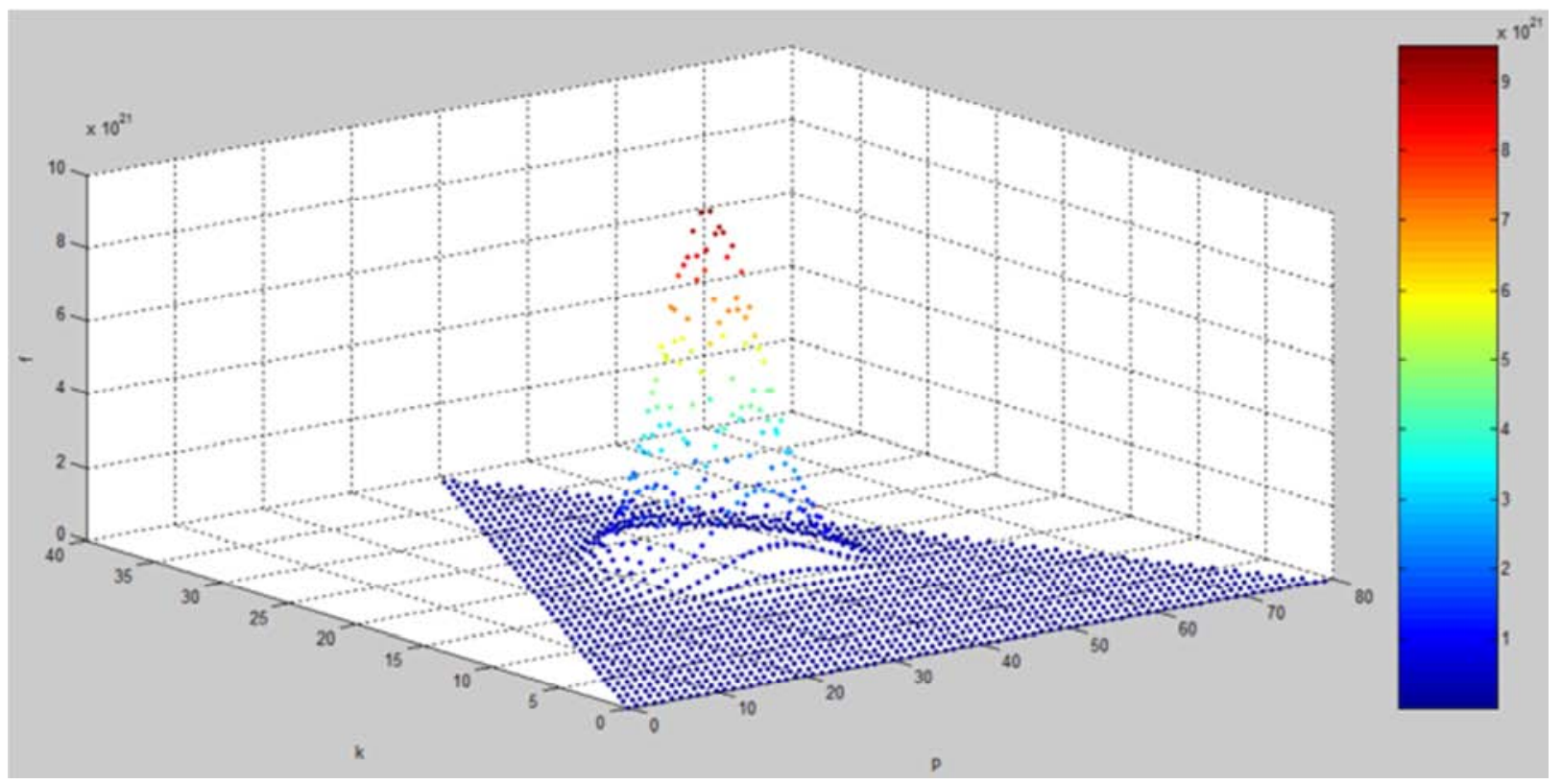

(a)

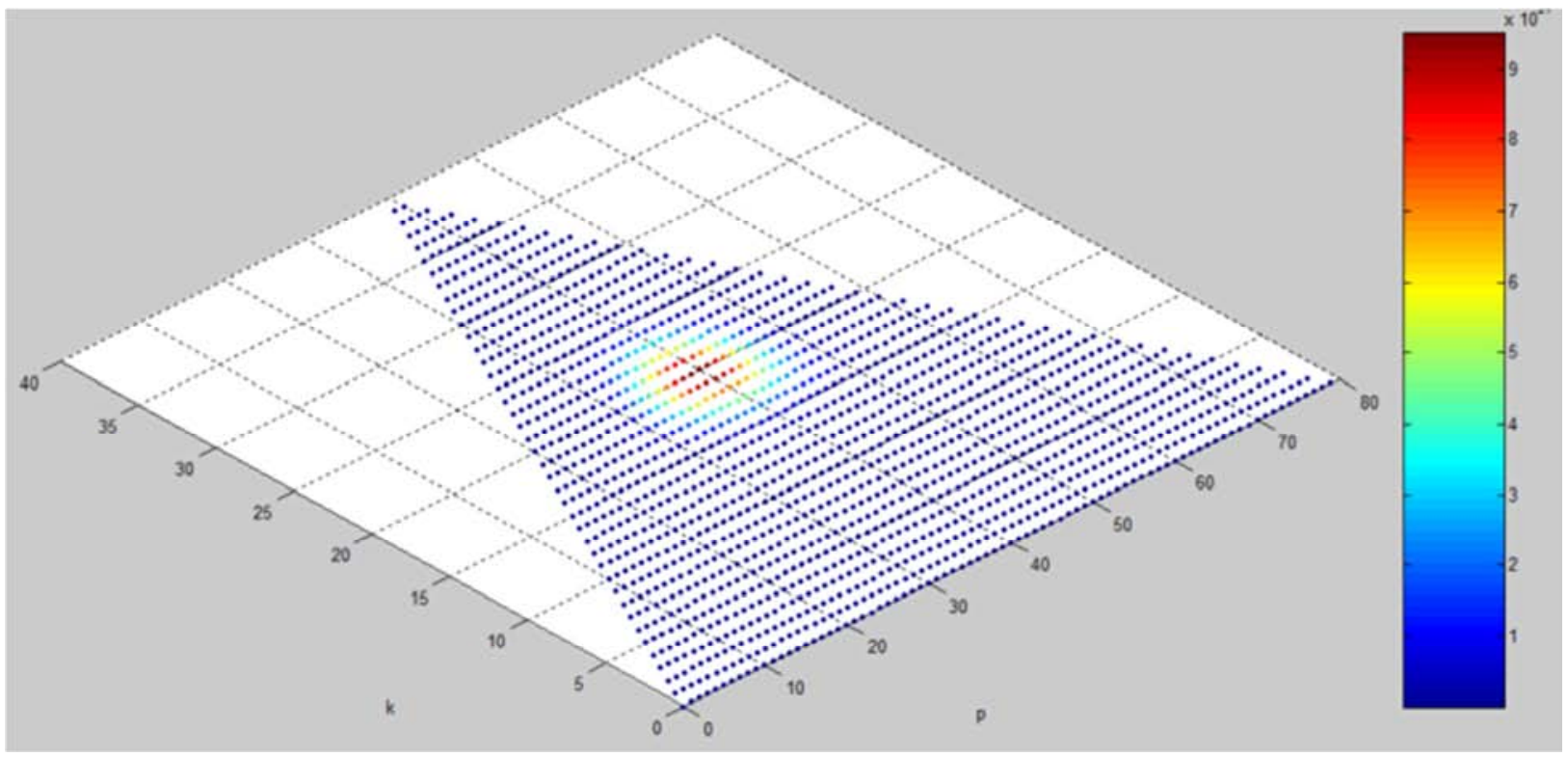

(b) 


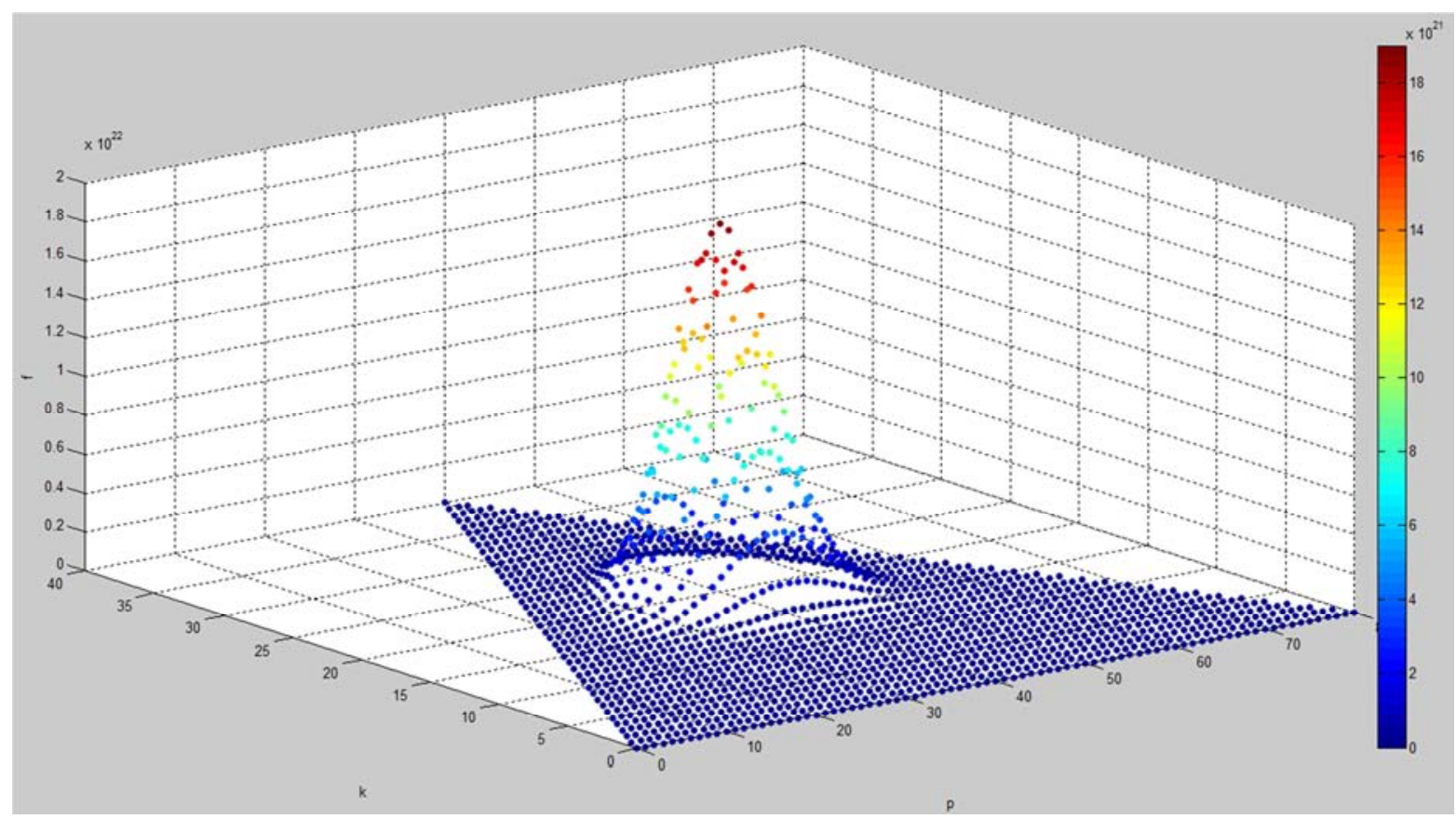

(c)

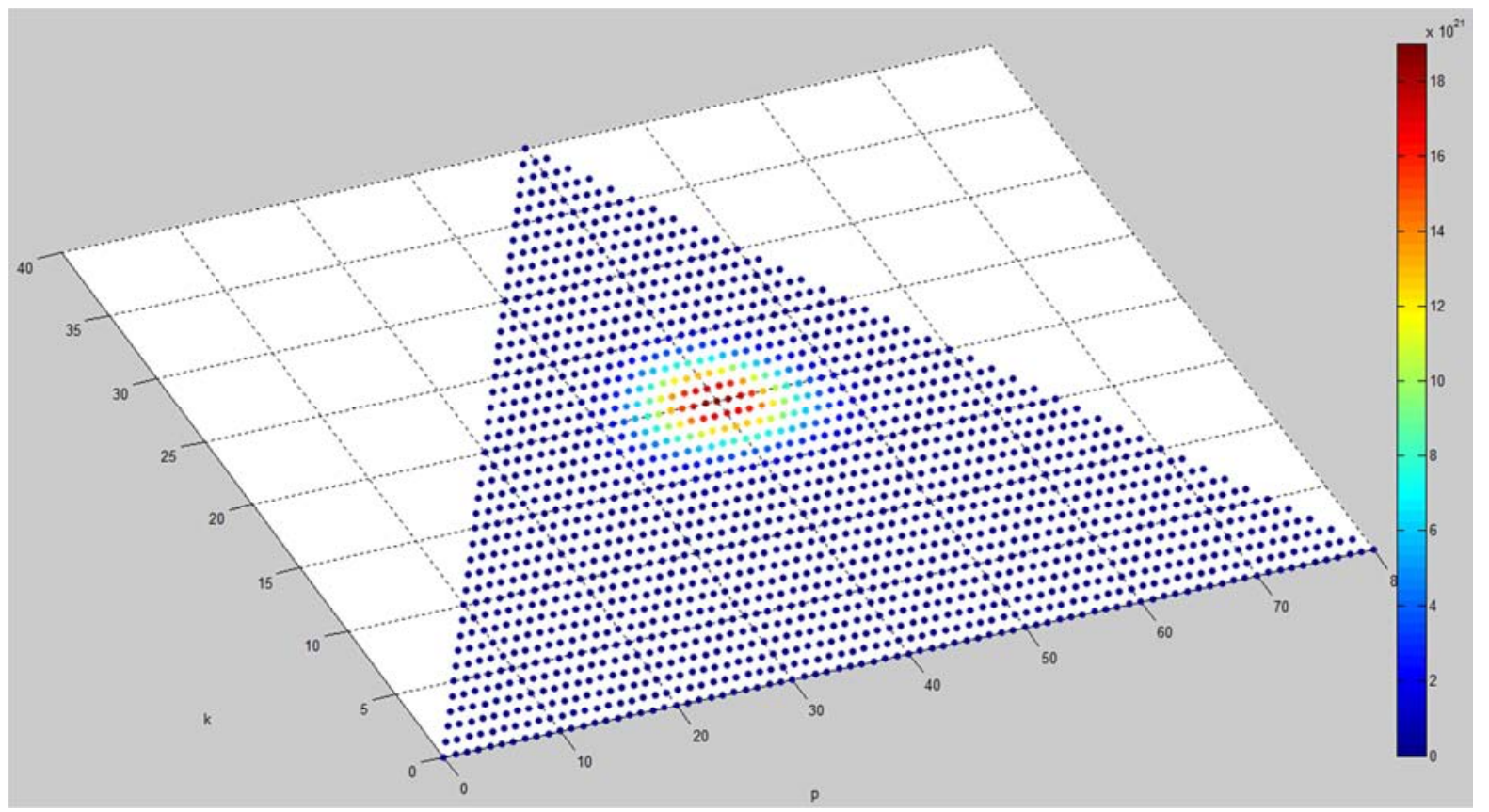

(d)

图9 二项式系数公式三维可视化图示 (a) $m=79$ 正视图 (b) $m=79$ 俯视图 (c) $m=80$ 正视图 (d) $m=80$ 俯视图。

由二项式系数公式: $f(m, p, k)=\left(\begin{array}{l}\mathrm{m}-\mathrm{p} \\ k\end{array}\right)\left(\begin{array}{l}p \\ k\end{array}\right)$, 推导出 其他公式及取值范围:

取 $0 \mathrm{pm} ; 0 \mathrm{kp} ; \mathrm{km}-\mathrm{p}$, 得出公式为 $f(m, p, k)=\left(\begin{array}{l}\mathrm{m}-\mathrm{p} \\ k\end{array}\right)\left(\begin{array}{l}p \\ k\end{array}\right)$, 取 $0 \mathrm{pm} ; 0 \mathrm{kp} ; \mathrm{k}>\mathrm{m}-\mathrm{p}$, 得出公式 为 $f_{2}(m, p, k)=\left(\begin{array}{l}\mathrm{k} \\ \mathrm{m}-\mathrm{p}\end{array}\right)\left(\begin{array}{l}\mathrm{m}-\mathrm{k} \\ \mathrm{m}-\mathrm{p}\end{array}\right)$;
取 $0 \mathrm{~km} ; 0 \mathrm{pk} ; \mathrm{km}-\mathrm{p}$, 得出公式为 $f_{3}(m, p, k)=\left(\begin{array}{l}\mathrm{m}-\mathrm{k} \\ p\end{array}\right)\left(\begin{array}{l}k \\ p\end{array}\right)$, 取 $0 \mathrm{~km} ; 0 \mathrm{pk} ; \mathrm{k}>\mathrm{m}-\mathrm{p}$, 得出公 式为 $f_{4}(m, p, k)=\left(\begin{array}{l}\mathrm{p} \\ m-k\end{array}\right)\left(\begin{array}{l}m-p \\ m-k\end{array}\right)$ 。

取 $m=79$ 以及 $m=80$, 根据所得公式作三维散点图示。 参阅图10(a)-(d)。

10(a)图取 $\mathrm{m}=79$, 图 $10(\mathrm{c})$ 取 $\mathrm{m}=80$, 都展示了不同数值 量化的分布情况。从图9(a)和图9(c)的单个峰波, 经过旋转 
反射，变为四个结构类似的峰波，并保留原有特征性质。 中心取值最大，依次向四周扩散，取值逐渐减小，四个峰 波仍都符合帕斯卡三角形法则。

通过观察图10(b)和图10(d), 取 $m=80$ 俯视图呈现矩形, 以中心对称, 并更明显的看出经过变换后的公式完全符合
反射旋转不变性的特征。两幅俯视图差异很小，特征并不 明显。 $\mathrm{m}$ 取值小的数值更易观察二项式公式及扩展模式的 数值分布情况。

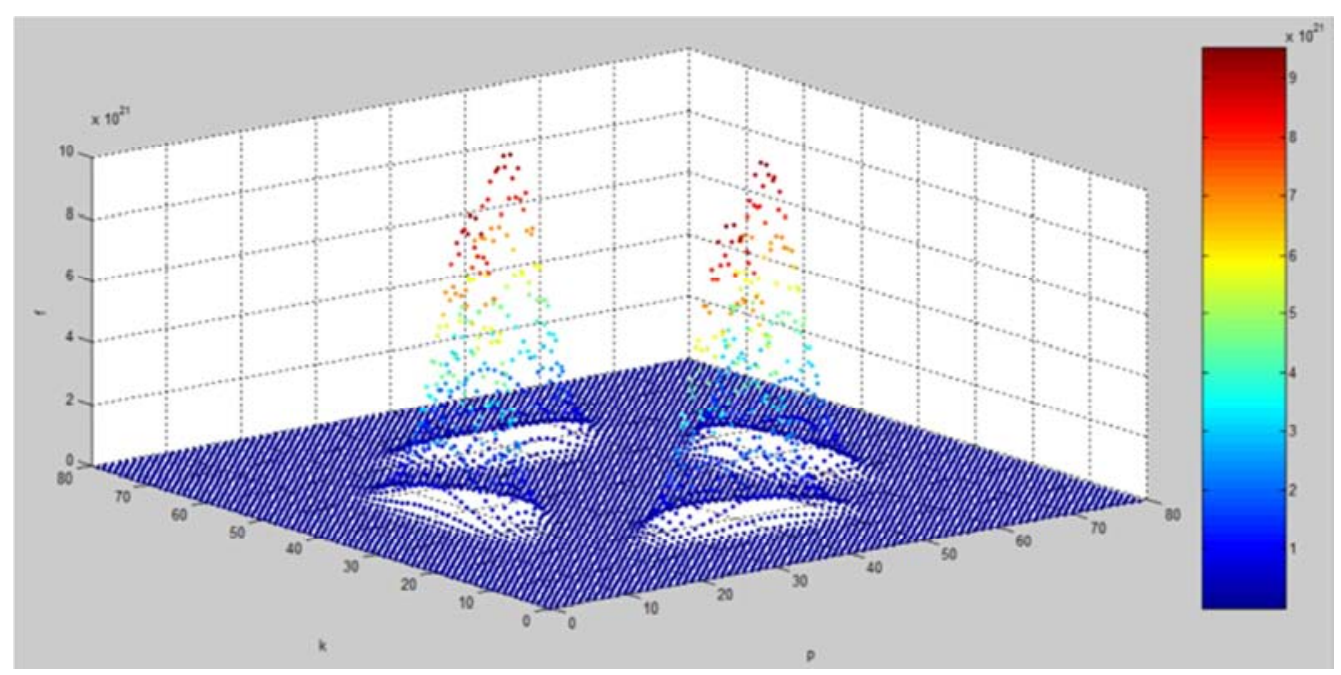

(a)

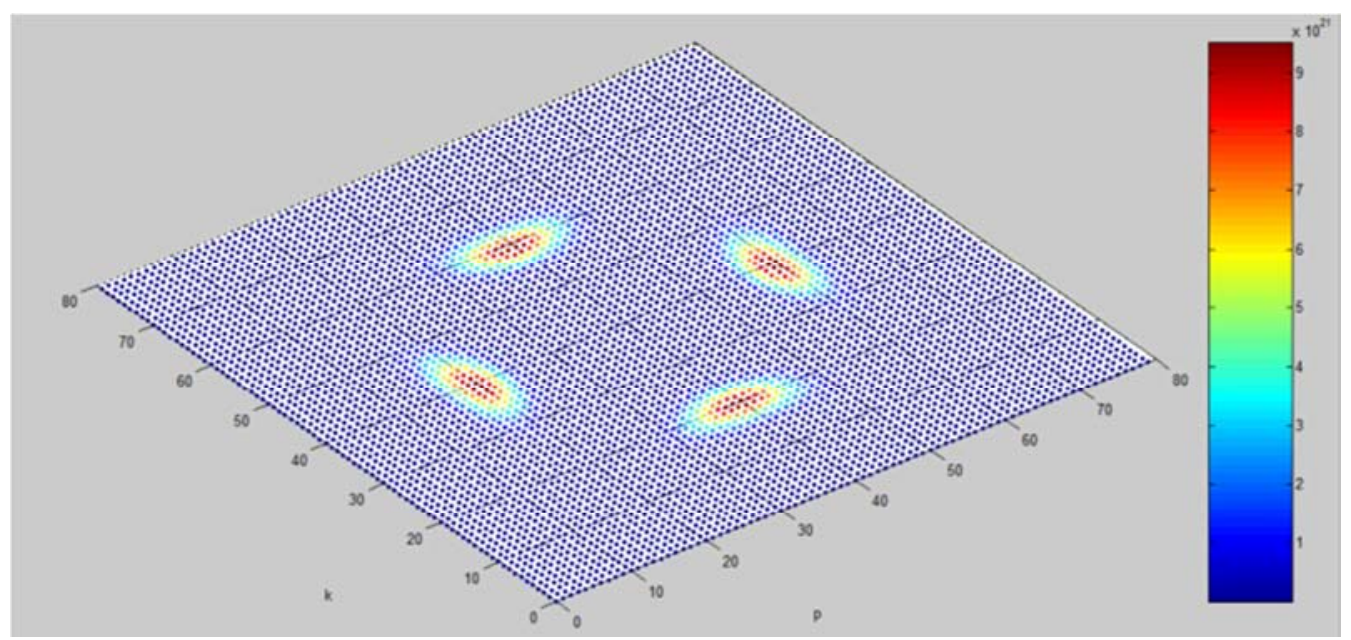

(b)

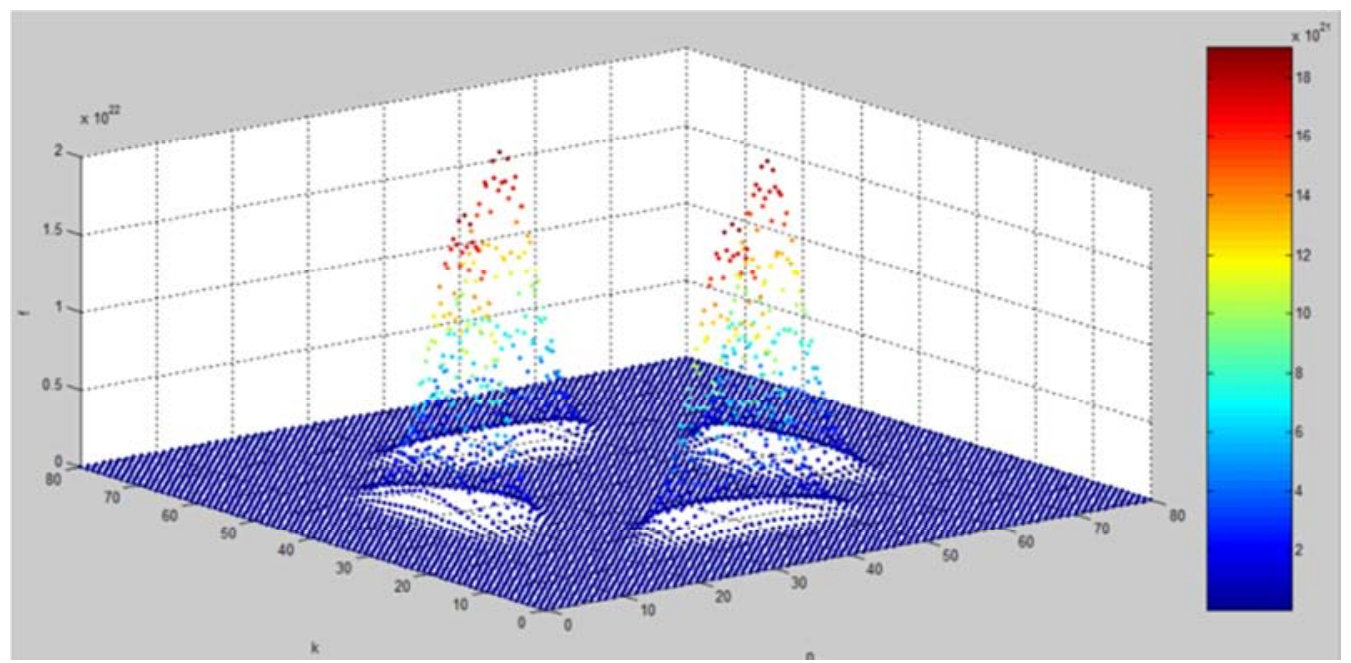

(c) 


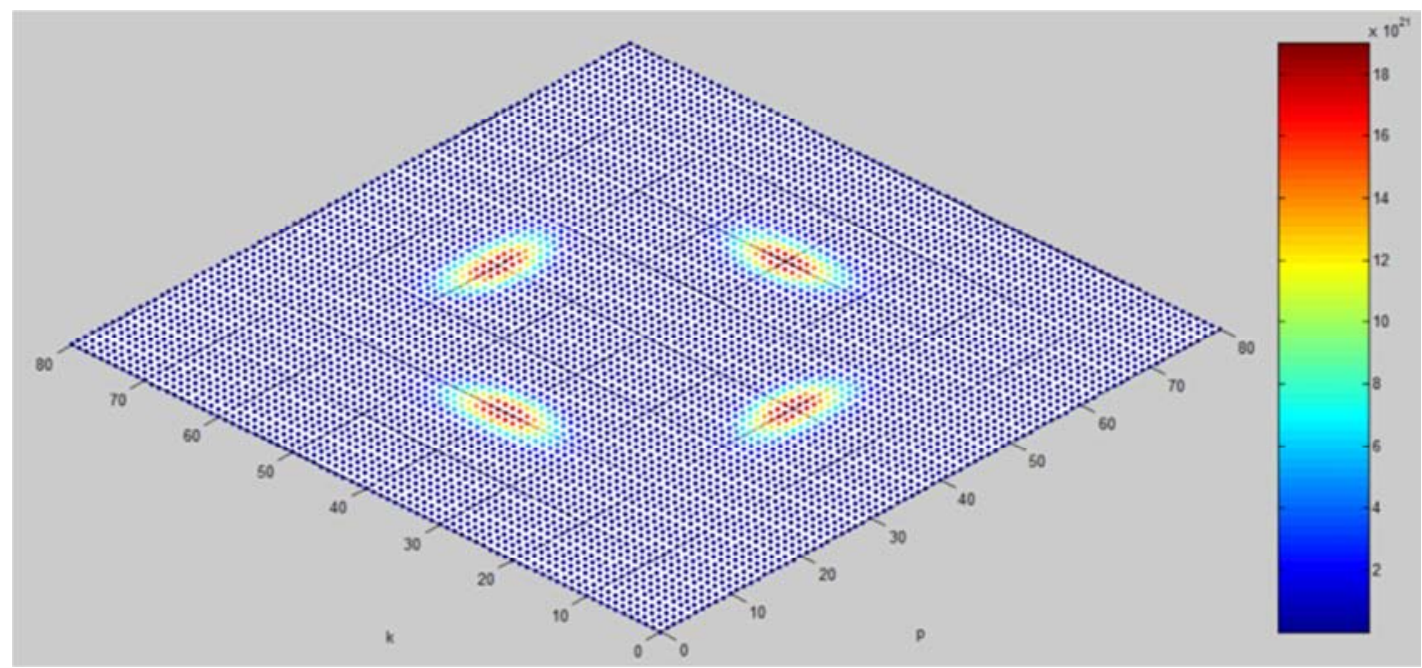

(d)

图10 基础二项式公式及扩展模式三维散点图示 (a)m=79正视图 (b)m=79俯视图 (c) $\mathrm{m}=80$ 正视图 (d)m=80俯视图。

\section{5. 结论}

利用基础二项式系数公式探索其扩展模式的系数分 布特性, 展现反射及旋转变换图示, 是一类新型的探索模 式。依据这些二项式系数公式, 可以更为方便地进行各种 组合分析和统计分布可视化。

所选择的基础二项式系数公式及其扩展性模式, 在奇 数和偶数的参数变换条件下, 形成可区分的反射及旋转特 性。根据2维直方图量化矩阵, 将对应的二项式系数公式 作三维可视化是有意义的。给出的系列变换实例展现了变 值测量形成新型二项式公式的潜在扩展能力, 为今后大数 据分析和复杂组合测量模式拟合等基础和应用型研究奠 定理论基础。

\section{致谢}

本项目由云南省量子保密通讯技术重大专项 (2018ZI002)和云南省海外高层次人才项目联合资助。

\section{参考文献}

[1] Brualdi,R.A. 组合数学 (原书第4版) [M]. 机械工业出版 社:2005。

[2] 庞兴梅. 组合变换在等式、多项式及简单图中的应用[D]. 天津：南开大学，2009。

[3] EvaPart-Enander. MATLAB 5手册[M]. 机械工业出版社: 2000 。
[4] Cleve Moler. Experiments with MATLAB[M]. 北京航空航 天大学出版社:2013。

[5] 尹社会. 利用伯努利系数表计算刚体转动惯量[R].高师理 科学刊, 2012, 32(3)。

[6] Knuth.D.E. 计算机编程的艺术 (第 4 卷: 组合算法,第 1 部 分)[M]. 国防工业出版社, 2011。

[7] Morgan.F. 几何测度论[M]. 世界图书出版公司, 2009。

[8] 吴军.数学之美[M]. 人民邮电出版社, 2012。

[9] Knuth.D.E. 计算机编程的艺术 (第1卷, 第3版) [M]. 国防 工业出版社, 2002。

[10] ZHENG Jeffrey Z. J. Variant Construction From Theoretical Foundations To Applications [M]. China: Springer, 2019: 39-50, 237-245.

https://link.springer.com/book/10.1007/978-981-13-2282-2

[11] ZHENG Jeffrey Z. J. A framework to express variant and invariant functional spaces for binary logic [J]. Front. Electr. Electron. Eng. China 5 (2), 163-172 (2010). Higher Educational Press and Springer.

[12] 赵熙强. Vandermonde卷积公式统一形式及其相应超几何 变换 $[J]$ 大连理工大学学报, 2000, 40(6)。

[13] 逯志宇. 基于对称旋转不变性的非圆相干分布源直接定位 算法[J]电子与信息学报, 2019,41(3)。

[14] 王庆平. 计量逻辑学中的反射变换 $[\mathrm{J}]$ 模糊系统与数学, 2018,32(6)。

[15] Kumar V. B. Efficient Computation of Binomial Coefficients Using Splay Trees [J]. International Journal on Data Science and Technology, 2016, 2 (1)。 